\title{
Evaluation of an IMU-Based Wearable Device for ACL Injury Research
}

\author{
Scott W. Kresie ${ }^{1}$, David A. Hawkins ${ }^{2,3,4 *}$ \\ ${ }^{1}$ Department of Mechanical and Aerospace Engineering, UC Davis, Davis, CA, USA \\ ${ }^{2}$ Department of Neurobiology, Physiology, and Behavior, UC Davis, Davis, CA, USA \\ ${ }^{3}$ Biomedical Engineering Graduate Group, UC Davis, Davis, CA, USA \\ ${ }^{4}$ Human Performance Laboratory, UC Davis, Davis, CA, USA
}

Received Date: April 15, 2021; Accepted Date: April 22, 2021; Published Date: May 02, 2021

*Correspondence Address: David Hawkins, College of Biological Sciences, Department of Neurobiology, Physiology, and Behavior, Room 196, Briggs Hall, University of California, One Shields Avenue, Davis, CA 95616, USA.

Tel: +1 530752 2748; Fax: Fax: (530) 752-6681; Email: dahawkins@ ucdavis.edu

\section{Abstract}

Anterior cruciate ligament (ACL) injuries are common, costly and have long-term health consequences. Despite decades of ACL injury research, ACL injury incidence remains high, highlighting the need for new approaches. We hypothesize that many non-contact ACL injuries result from an overuse, fatigue failure mechanism and that advances in wearable technology provide an exciting opportunity to obtain data needed to test this hypothesis. In this study, we created and evaluated an inertial measurement unit (IMU) based wearable device to collect a clinically relevant metric of ACL loading events from athletes in the field. The wearable device measures relative anterior tibial acceleration (RATA), a surrogate measure of ACL loading defined as the relative acceleration between a point on the proximal tibia and a point on the distal femur. The device was evaluated using a mechanical test system designed to simulate human leg movement, and then used to quantify peak RATA profiles for two participants performing six common athletic movement tasks associated with relatively low and high levels of ACL loading. The wearable device successfully tracked in-vivo the frequency and magnitude of ACL loading events and thus provides a tool to acquire data needed to test fatigue failure mechanism theories of ACL injury.

Keywords: Anterior tibial acceleration; Loading history; Overuse injury; Wearable sensors

\section{Introduction}

Non-contact Anterior Cruciate Ligament (ACL) injuries make up $70 \%$ of all reported ACL injuries [1, 2] and are an important clinical problem affecting many people and having large financial and quality of life implications. Despite decades of research, ACL injuries continue to be a problem and new strategies to prognose and prevent these injuries are warranted. Non-contact ACL injuries are commonly theorized to be acute injuries caused by a single catastrophic overload of the ligament that occurs an estimated $40-50 \mathrm{~ms}$ after the foot makes contact with the ground $[3,4]$. However, the single catastrophic overload theory fails to explain why an athlete can perform the same athletic movement such as a jump landing hundreds of times without injury, but suddenly rupture the ACL when the movement is performed one more time. Consistent with these results is a fatigue failure mechanism that reduces the ligament's ultimate tensile strength (UTS) over time. Evidence from ex-vivo animal ligament studies [57] show that repetitive loading at a sub-failure magnitude causes microdamage in the ACL and reduced UTS and stiffness. Lipps, Wojtys, and Ashton-Miller (2013) found human cadaveric ACLs failed when subjected to repetitive loading simulating realistic knee compression forces, torques, ACL strain rates and sub-maximal ACL strains representative of common jump landing movements [8]. Molecular, nanoscopic, and microscopic imaging of ACLs from cadaveric knees subjected to realistic repetitive loading provide evidence of structural damage in the collagen fibrils and fibers that show the same damage signatures as ACL explants from patients who suffered an ACL injury [9]. Therefore, investigation of a fatigue failure mechanism of ACL injury is warranted. Traditional lab-based ACL injury research methods are insufficient to address non-contact ACL injury fatigue failure mechanisms. These methods address acute overload mechanism of ACL injury by identifying specific movement patterns that are associated with high ACL loading. However, these traditional methods cannot address the fatigue failure theory of ACL injury since loading history is not collected. Furthermore, these methods do not collect loading data in ecologically valid environments. To properly investigate the role cyclic subfailure loading plays in ACL injury, tools need to be developed that can measure ACL loading history in an ecologically valid environment. Wearable devices offer a means to measure relative anterior tibial acceleration (RATA), a theorized surrogate metric of ACL loading, throughout physical activities in an ecologically valid environment. RATA is defined as the relative acceleration between tibial and femoral origins, as defined by Grood and Suntay [10], in the tibial anterior direction. Peak RATA correlated with ACL strain in a cadaveric simulated vertical jump landing study $(r=0.79)$ [11]. Wearable accelerometer devices and inertial measurement units (IMUs), which integrate accelerometers, gyroscopes, and sometimes magnetometers, provide an exciting opportunity to quantify surrogate metrics of ACL loading, specifically RATA. In this study, we developed and tested an inertial measurement unit (IMU) based wearable device to quantify RATA throughout an athletes' normal training.

\section{Methods}

\section{RATA Calculation}

The theory for calculating RATA using two IMUs (one worn on the thigh, one worn on the shank) is based on fundamental dynamics principles of rigid-bodies (i.e. the acceleration of any point on a rigid body can be calculated if the acceleration of another point on the rigid body along with the position vector between the two points, the angular velocity, and angular acceleration of the body are known). RATA was defined as the relative acceleration between the 
tibial and femoral origins, as defined in Grood and Suntay [10], in the tibial anterior direction. Theoretically, data from IMU sensors (acceleration and angular velocity) positioned arbitrarily relative to limb segment origin points (Figure 1) can be used with controlled and specific leg flexion and extension motions (Nazarahari et al. (2019) [12] and Seel et al. (2014) - [13]) to determine the direction cosine matrix (DCM) relating the IMU coordinate system and the limb coordinate system, and the position vector from the segment origin to the IMU accelerometer origin. Subsequently, RATA can be determined for any limb movement using the IMU sensor data, IMU to segment DCMs, and IMU to segment origin position vectors. Calibration procedures and software were established to operationalize the theoretical RATA calculations for practical applications.

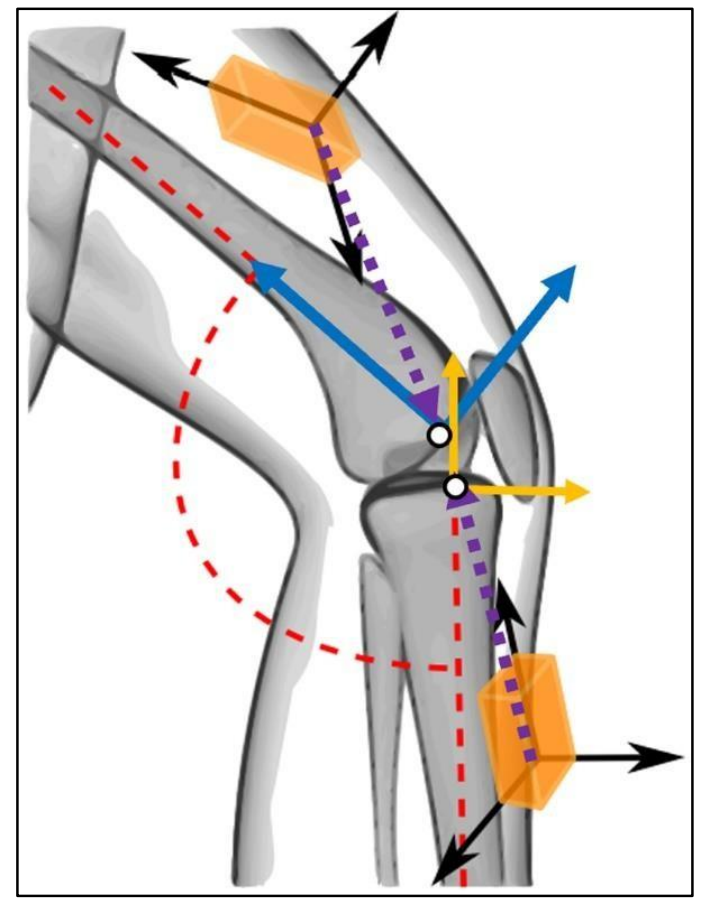

Figure 1: An illustration (adapted from Seel et al. [13]) showing the arbitrary orientation of each IMU's reference frame (black arrows) and position relative to the segment origin when worn on the thigh and shank. The dotted position vectors illustrate the position of the tibial and femoral origins (white points) relative to the IMUs. The anatomical anterior and longitudinal directions (denoted by solid arrows) for each segment are shown.

\section{Mechanical Leg Testing Methods}

A mechanical human-leg analog test rig (Figure 2), was fabricated and used to evaluate the RATA algorithm and calibration procedures that operationalized the theoretical RATA calculations. The rigid mechanical system was operated with and without a 1-inch-thick layer of ballistics gel on the thigh segment and a 1/8-inch-thick layer on the shank segment to simulate human leg soft-tissue. The mechanical model experiment served as a checkpoint to ensure the RATA algorithm performed properly prior to human application. The experiment also provided an opportunity to improve the algorithm by identifying and mitigating the soft-tissue effects on the IMU data and RATA calculation.

Dropping the mechanical system to simulate a knee loading event elicited peak RATA values ranging from 10 to $55 \mathrm{~m} / \mathrm{s}^{2}$. RATA was measured simultaneously using the wearable IMU device and an optical motion capture system (Innovision Systems Inc.) and values compared.

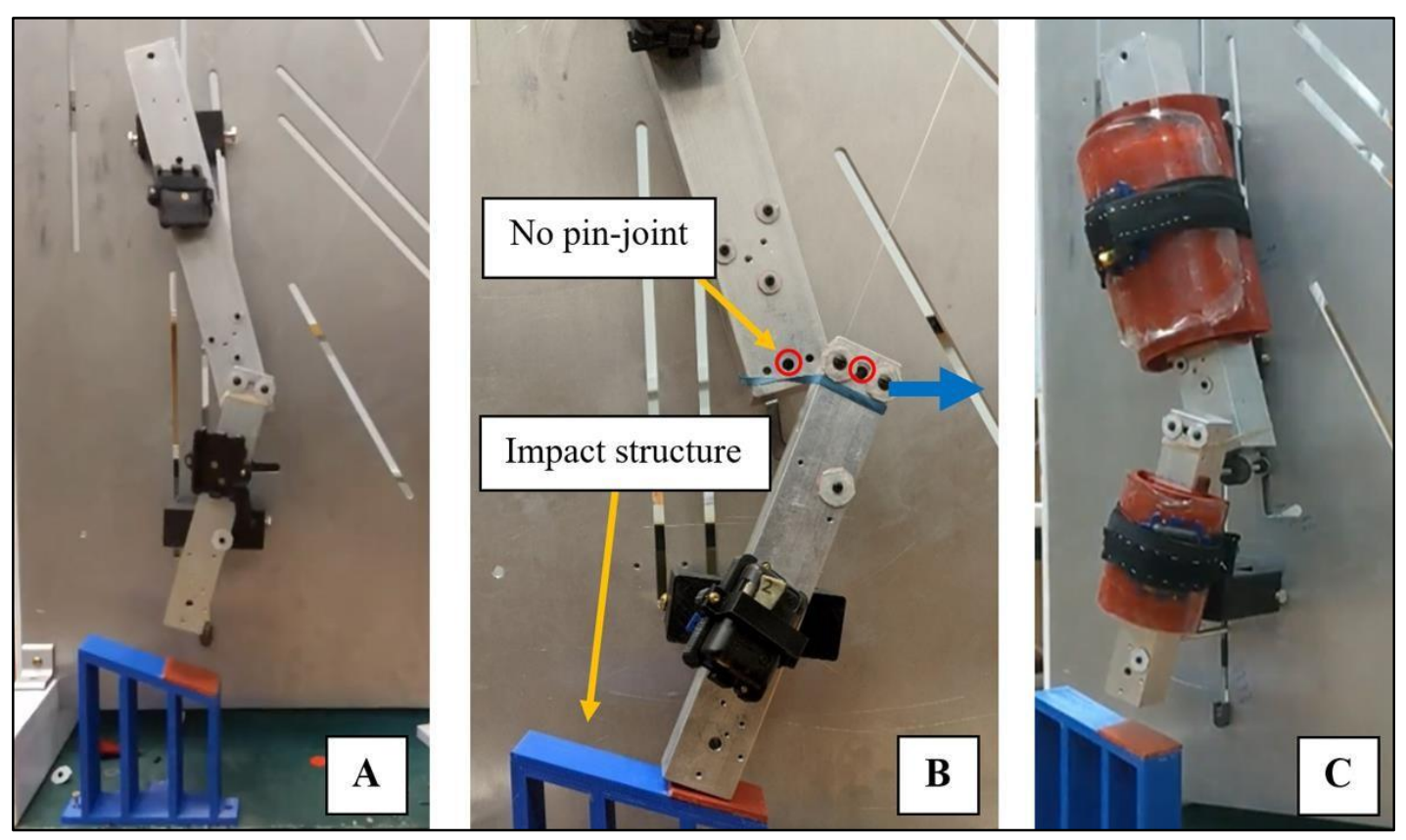

Figure 2: (A) The mechanical leg model in free-fall configured for eliciting RATA. A combination of slots, pins, and rollers facilitate the planar motion. High contrast motion capture markers on both segments are screwed into known locations in each segment. (B) The mechanical thigh and mechanical shank are not connected by a pin-joint and can move independently of each other allowing for RATA. When the mechanical shank collides with the impact structure the shank accelerates horizontally (indicated by the horizontal arrow). A component of the acceleration in the horizontal direction is in the anterior direction of the tibia. (C) The system fit with layers of ballistic gel to simulate human leg soft-tissue. 


\section{Human Testing Methods}

Two male, recreational athletes, performed six movement tasks, three of which were anticipated to be relatively low ACL loading and three were anticipated to involve relatively high ACL loading while wearing the device. Both participants adhered to COVID-19 mask and physical distancing guidelines throughout testing. The study was approved by the University of California, Davis Institutional Review Board. Participants wore form-fitting spandex pants that featured custom velcro attachment sites to secure one IMU on the thigh and one on the shank portion of the right pant-leg (Figure 3). The NGIMU ( $\mathrm{x}$-io Technologies Limited, Bristol, United Kingdom) was selected for the wearable device. These IMUs logged tri-axial acceleration $( \pm 16 \mathrm{~g})$, angular velocity $( \pm$
$2000 \%$ ), and 3D orientation (estimated with an on-board sensor fusion algorithm) to a microSD card at $400 \mathrm{~Hz}$. The IMU data streams were synchronized by cross-correlating acceleration signals during a manual calibration and elastic straps were used to secure the IMUs to the thigh and shank. The participants performed the calibration procedures (previously validated by the mechanical testing system) to transform sensor data to the anatomical reference frame of each body segment and to estimate the location of the tibial and femoral origin (approximated as the knee-joint-center) relative to the accelerometer of each IMU. The participants then performed a series of activities (Figure 3): walking, jogging, vertical jump, jump-stop, run and cut, and sprint to backpedal. A total of 10 to 15 trials were completed by each participant for each activity.
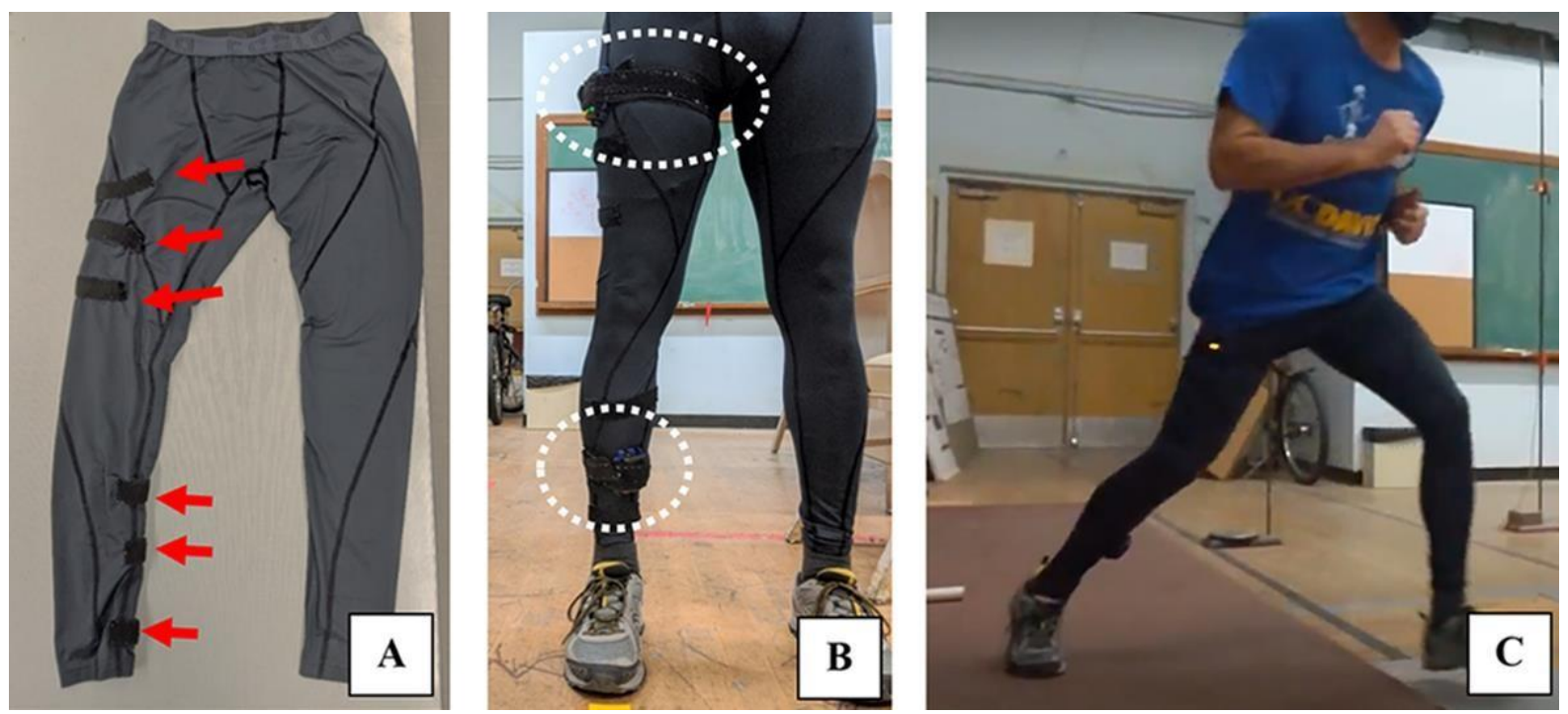

Figure 3: (A) Spandex form-fitting pants with velcro attachment sites, indicated by arrows, for securing the IMU holder and strap. The attachment sites that most securely held the IMUs to the leg were identified for each participant. (B) IMUs were mounted on the lateral upper thigh and medial shank with custom elastic velcro straps. (C) Screenshot of video taking during a run-and-cut trial. The participant sprinted forward several strides and cut at a 45-degree angle, planting the leg with sensors attached. This was one of six different movements performed by each participant.

Signals from each axis of each IMU were filtered with a $4^{\text {th }}$-order Butterworth filter with a cutoff frequency determined by the methods described by Yu et al. (1999) [14]. Peak RATA was calculated within a $100 \mathrm{~ms}$ time window after right foot ground contact. $100 \mathrm{~ms}$ was a conservative time window based on peak RATA and ACL strain occurring 51 to $76 \mathrm{~ms}$ after impact in the Mclean et al. simulated jump landing cadaver leg study [11]. Soft-tissue differences experienced by the IMUs worn on the thigh and shank caused phase lag between the tibial and femoral origin acceleration signals. To address the phase lag, peak RATA values were calculated using paired inflection points between the anterior acceleration signal of the tibial and femoral origin that produced the maximum positive difference (or minimum negative difference) of anterior acceleration (Figure 4).

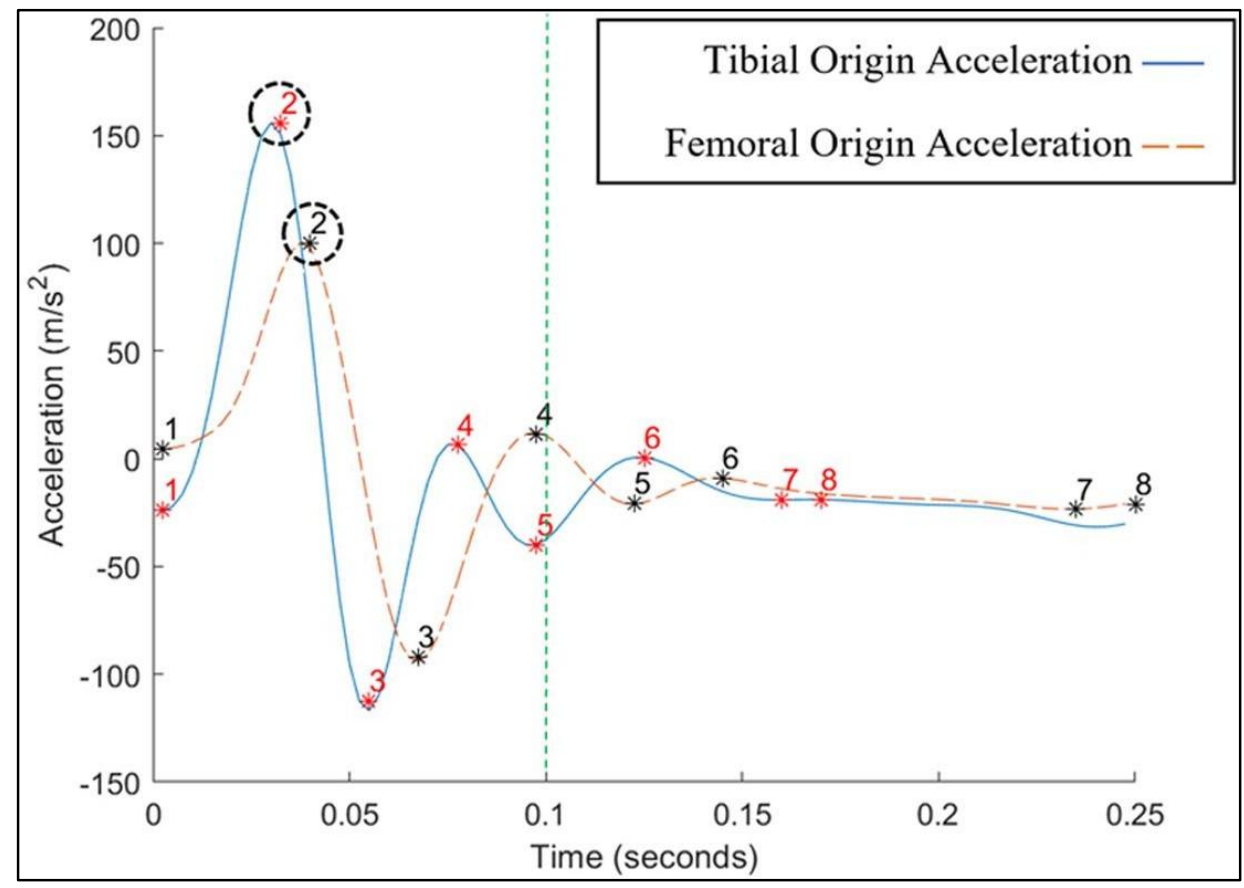

Figure 4: Anterior acceleration signals (tibia frame) of the tibial (solid line) and femoral (dashed line) origins for a run and cut trial. Paired inflection points are marked with the same number. Only the first 0.1 seconds after impact (dashed line) were used to calculate a peak RATA, which for this example would be the difference between circled inflection points. 
The primary analysis consisted of determining RATA thresholds that separated the trials corresponding to the less intense activities (walking, jogging, and vertical jump) from the more intense activities (jump-stop, run and cut, and sprint to back pedal). If such thresholds existed, then it seemed logical that this approach could be refined to track peak RATA history and identify values associated with ACL injury. The recorded peak RATA for each trial was normalized by each participant's mean peak RATA during walking. Subjectspecific thresholds were identified such that the maximum number of low intensity activity trials had a normalized peak RATA below the threshold and such that the maximum number of high intensity activity trials had a normalized peak RATA above the threshold.

\section{Results}

\section{Mechanical Leg Testing Results}

There was good agreement between RATA calculated using the IMU device and the optical system for the rigid mechanical leg, but less agreement for the tests simulating soft tissue. IMU-measured peak RATA was within $\pm 20 \%$ of the optical motion capture measurement for $91 \%$ of trials without ballistic gel and $60 \%$ of the trials with Ballistic gel. Due to the soft-tissue on the mechanical thigh being relatively thick and the soft-tissue on the mechanical shank being relatively thin (more like a rigid-body) there was a phase lag between the femoral and tibial acceleration used to calculate RATA. Phase lag was determined by comparing analyzed acceleration signals derived from IMU data (influenced by the ballistic gel soft-tissue) to the equivalent signals derived from motion capture (markers were screwed into rigid bodies and were not influenced by the ballistic gel soft-tissue). Modifying the RATA algorithm to compensate for phase lag, the percentage of trials that had IMU-measured peak RATA within $\pm 20 \%$ of the optical motion capture measurement increased from $60 \%$ to $83 \%$. Phase lag compensation was incorporated into the data processing associated with human testing.

\section{Human Testing Results}

For participant \#1 and \#2 the normalized peak RATA thresholds were 2.46 and 2.52, respectively (Figure 5). For participant $\# 1,94 \%$ of the low intensity activity trials fell below the threshold while $93 \%$ of the high intensity activity trials fell above the threshold. For participant \#2, 93\% of the low intensity activity trials fell below the threshold while $67 \%$ of the high intensity activity trials fell above the threshold.

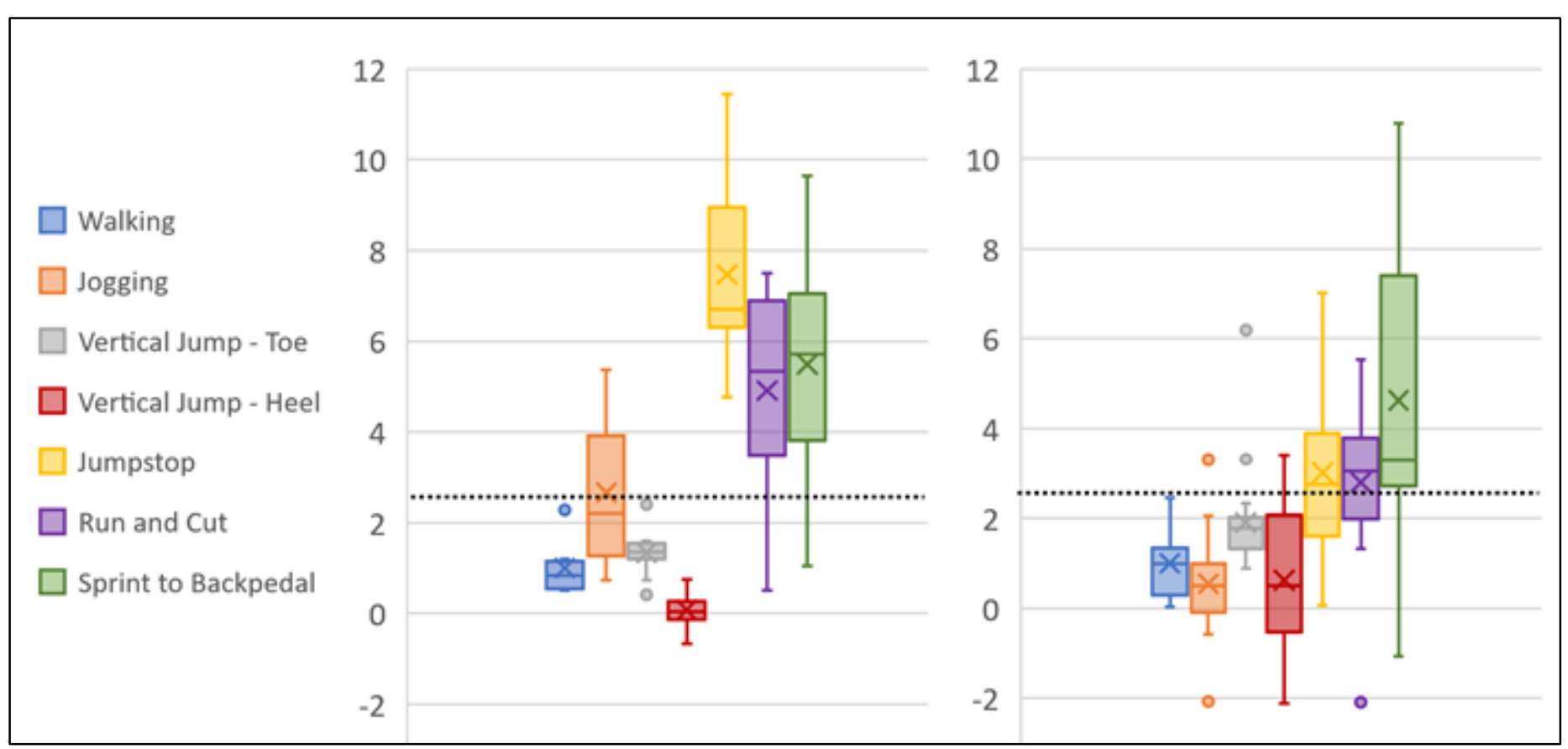

Figure 5: Box and whisker plots displaying the distribution of normalized peak RATA for all trials of each activity for each participant (participant \#1 - left, participant \#2 - right). The vertical jump activity had two distinct contact events. The toe contacted the ground first followed by the heel. Peak RATA values were calculated for both events for each trial. The horizontal line and cross in each box represent the median and mean, respectively. The black dashed lines represent the normalized peak RATA threshold identified for each participant.

\section{Discussion}

We hypothesize that many non-contact ACL injuries result from an overuse, fatigue failure mechanism and that advances in wearable technology provide an exciting opportunity to obtain data needed to test this hypothesis. In this study, we developed and tested an IMU-based wearable device to quantify RATA, a theorized surrogate metric of ACL loading, throughout an athlete's normal training. One key assumption is that RATA is an effective surrogate measurement of ACL strain or loading. This assumption is based on the results of a cadaveric study [11] that showed a positive correlation between RATA and ACL strain. The framework for a wearable device to track injury related events over time already exists in instrumented football helmets. The Head Impact Telemetry System (HIT System) has been used to monitor head impacts [15] during training and competition to better identify concussions in the field and to develop models that analyze the conditions that lead to concussion $[16, \mathbf{1 7}]$. The HIT System demonstrates the feasibility of using IMUs to track clinically relevant metrics that correlate with injury and provided the motivation to design and test a wearable, IMU- based, RATA measurement device to track clinically relevant metrics of ACL loading.

A limitation was that RATA data were collected from only two participants in human testing. The goal was to collect data from a large group of participants, but this was not practical or ethical during the COVID-19 world pandemic. Future work should involve testing many subjects over multiple days to determine if the RATA results are reproducible within a single subject and across subjects. Rigid-body dynamics principles indicated RATA could be calculated using two IMUs. The mechanical human-leg system operationalized these principles in a practical setting and demonstrated the potential utility of an IMU-based wearable device. The mechanical tests with ballistic gel identified a soft-tissue induced phase lag between acceleration signals derived from the thigh-worn and shankworn IMUs. This finding informed IMU data analysis methods to compensate for the phase lag in the human participant study.

The results from the human participant study were encouraging despite the small sample size. Consistency in peak RATA measurements for each activity allowed identification of a 
peak RATA threshold that separated the majority of lower intensity (walking, jogging, vertical jump) activity trials from the higher intensity activity trials (jump stop, run and cut, and sprint to backpedal). When the peak RATA data were normalized by the mean peak RATA during walking both participants had nearly the same threshold value (2.46 and 2.52) (Figure 5). Future work featuring larger sample sizes should investigate if the threshold is consistent between participants or between recording sessions for the same participant. If so, the normalization strategy could be used to control for differences in soft-tissue at the attachment site, strap-tightness, and sensor placement between participants or between recording sessions. The wearable device was found to yield consistent peak RATA measurements within each activity allowing identification of a threshold between relatively high and low ACL loading movements.

The next major advances in ACL injury prevention research will likely come from identifying clinically relevant metrics associated with ACL loading that can be measured in ecologically valid environments using wearable technology. The relationships between RATA history, sub-failure ACL damage and increased ACL injury risk is not known, however, the IMU-based wearable developed in this project provides a foundation and a tool to explore these relationships in the future.

\section{Conflict of Interest Disclosure: None}

\section{References}

1. Lubowitz JH, Appleby D (2011) "Cost-Effectiveness Analysis of the Most Common Orthopaedic Surgery Procedures: Knee Arthroscopy and Knee Anterior Cruciate Ligament Reconstruction." Arthroscopy: The Journal of Arthroscopic \& Related Surgery 27: 1317-22.

2. Griffin LY, Agel J, Albohm MJ, Arendt EA, Dick RW, et al. (2000) "Noncontact Anterior Cruciate Ligament Injuries: Risk Factors andPrevention Strategies." J Am Acad Orthop Surg 8: 141-150.

3. Koga H, Nakamae A, Shima Y, Iwasa J, Myklebust G, et al. (2010) "Mechanisms for Noncontact Anterior Cruciate Ligament Injuries: Knee Joint Kinematics in 10 Injury Situations from Female Team Handball and Basketball”. Am J Sports Med 38: 2218-2225.

4. Krosshaug T, Nakamae A, Boden BP, Engebretsen L, Smith G, et al. (2007) "Mechanisms of Anterior Cruciate Ligament Injury in Basketball: Video Analysis of 39 Cases." Am J Sports Med 35: 359-367.

5. Thornton GM, Schwab TD, Oxland TR (2007) "Fatigue is More Damaging than Creepin Ligament Revealed by Modulus Reduction and Residual Strength.” Ann Biomed Eng 35: 1713-1721.

6. Panjabi MM, Yoldas E, Oxland TR, Crisco JJ, "Subfailure injury of the rabbit anterior cruciate ligament." Journal of Orthopaedic Research 14: 216-222.

7. Provenzano PP, Heisey D, Hayashi K, Lakes R, Vanderby $R$ (2002), "Subfailure damagein ligament: a structural and cellular evaluation." Journal of Applied Physiology 92: 362-371.

8. Lipps DB, Wojtys EM, Ashton-Miller JA (2013) "Anterior Cruciate Ligament Fatigue Failures in Knees Subjected to Repeated Simulated Pivot Landings.” Am J Sports Med 41: 1058-1066.

9. Chen J, Kim J, Shao W, Schlecht SH, Baek SY, et al. (2019) "An Anterior Cruciate Ligament Failure Mechanism.” Am J Sports Med 47: 2067-2076.

10. Grood ES, Suntay WJ (1983) "A Joint Coordinate System for the Clinical Description of Three-Dimensional Motions: Application to the Knee.” J Biomech Eng 105: 136-14.

11. McLean SG, Oh YK, palmer ML, Lucey SM, Lucarelli DG, et al. (2011) "The Relationship Between Anterior Tibial Acceleration, Tibial Slope, and ACL Strain During a Simulated Jump Landing Task." The Journal of Bone and Joint Surgery-American 93: 1310-1317.

12. Nazarahari M, Noamani $\mathrm{N}$, Ahmadian $\mathrm{N}$, Rouhani $\mathrm{H}$ (2019) "Sensor-to-body calibrationprocedure for clinical motion analysis of lower limb using magnetic and inertial measurement units." Journal of Biomechanics 85: 224229.

13. Seel T, Raisch J, and Schauer T (2014) IMU-Based Joint Angle Measurement for Gait Analysis Sensors 14: 68916909.

14. Yu B, Gabriel D, Noble L, An KN (1999) "Estimate of the Optimum Cutoff Frequency for the Butterworth LowPass Digital Filter...” Journal of Applied Biomechanics 15: 318-329.

15. O'Connor KL, Rowson S, Duma SM, Broglio SP (2017) "Head-Impact-MeasurementDevices: A Systematic Review. J Athl Train 52: 206-227.

16. Campolettano ET, Gellner RA, Smith EP, Bellamkonda S, Tierney CT, et al. (2020) Development of a Concussion Risk Function for a Youth Population Using Head Linear and Rotational Acceleration." Ann Biomed Eng 48: 92103.

17. Broglio SP, Lapointe A, O’Connor KL, McCrea M (2017) "Head Impact Density: A Model To Explain the Elusive Concussion Threshold. Journal of Neurotrauma 34: 26752683 